and words used in the guide and to suggest alternative wording for the prompts they felt did not feel comfortable to say or hear.

Results A number of key themes emerged from the workshops - the language felt too stiff and formal, the tone was paternalistic, the guide left people feeling talked at and not partnered with, the language needed to be simplified, the doctor should not be "worried".

The input and suggestions from the workshops were synthesised and an Aotearoa version of the Guide developed.

\section{P04 KIA KORERO : LET'S TALK - NEW ZEALAND ACP CAMPAIGN 2019}

${ }^{1} \mathrm{~L}$ Manson*, ${ }^{1} \mathrm{C}$ O'Leary, ${ }^{2} \mathrm{~A}$ Blank, ${ }^{1} \mathrm{~L}$ Price. ${ }^{1}$ Health Quality Safety Commission, Wellington, New Zealand; ${ }^{2}$ Oranui, Auckland, New Zealand

\subsection{6/spcare-2019-ACPICONGRESSABS.96}

The first public facing advance care planning campaign in New Zealand will feature the diverse voices of people at different stages of life and wellness, incorporating $\mathrm{M} \square$ ori, Pacific, Asian, Pakeha (European) and LGBGTQI communities. It begins with the story of kuia (elder) M $\square$ ori woman, Keri Kaa who is in the last phase of life.

The campaign, Kai Korero, Let's Talk, recognises the indigenous people as tangata whenua (the first people of the land), and aims to reflect the diversity of the communities we work with.

It will be launched with digital stories from February to April 2019, through social media such as Facebook, Instagram and Twitter. This is in response to our stakeholder engagement with $\mathrm{M} \square$ ori communities who have adapted quickly to digital resources. $\mathrm{M} \square$ ori is an oral culture and $\mathrm{p} \square$ raka $\square$ (storytelling) is an essential part of knowledge sharing.

It is also likely that print elements such as posters will be developed, and there will be a strong public relations component focusing particularly on $\mathrm{M} \square$ ori and Pacific media.

We hope the campaign will inspire people to have a conversation with their loved ones and their doctors about what matters to them if they become unwell, to discuss what kind of care they would or wouldn't want and to write down what is important to them.

\section{P05 IMPLEMENTATION OF A MULTI-MODALITY EDUCATION PROGRAM IN AUSTRALIA}

D Detering*, M Pigott, M Wills, M Nolte. Austin Health, Heidelberg, Australia

10.1136/spcare-2019-ACPICONGRESSABS. 97

Background Despite the acknowledged importance of advance care planning (ACP), a barrier to uptake is appropriately skilled clinicians to have conversations. A literature review showed limited availability of education resources that considered the Australian context. This implementation project involved the development of education resources that facilitated scaffolding of learning, from novice to expert, and included online modules, clinician workshops and train the trainer workshops as part of a standardised program of education in ACP.
Methods Nine online modules, education resources to enable clinicians to practice ACP discussions in workshops and training for facilitators to implement their own workshops were developed.

Results From July 2017 to June 2018, 2656 people were registered on the learning management site and 1541 completed at least one online module. Feedback from the online evaluation identified that $99 \%$ of 4262 people rated their likelihood of recommending the module to colleagues as $\geq 5$ out of 10 . Seventy percent of the 144 people who attended the clinician workshops in the 12 -month period specifically identified communication with patients and colleagues as the key area of learning for implementation. From 16 people who attended the two facilitator's workshops 6 have accessed the education resources and facilitated their own workshops.

Conclusion This program considered the implementation of ACP education using a framework for learners to scaffold their knowledge. The suite of education resources provides a sustainable program of education by encouraging development of skills to the expert facilitator level. There is clearly a demand and interest in multi-modality learning.

\section{P07 ADVANCE CARE PLANNING REDUCES HEALTHCARE UTILISATION AND SAVES COST}

CJ Ng*, ML Ong, F Li, JYH Low. Khoo Teck Puat Hospital, SINGAPORE, Singapore

\subsection{6/spcare-2019-ACPICONGRESSABS.98}

Background Advance Care Planning (ACP) is an important conversation that patients have with their care team to understand their medical condition and establish goals of care. Few studies, however, have investigated its impact on hospital resources. This study aims to determine the association between ACP and its effect on healthcare utilisation and cost.

Methods 1343 patients from a tertiary hospital in Singapore completed either a General ACP or ACP - Preferred Plan of Care (PPC) from January 2013 to December 2017. Healthcare utilisation data was studied for each group preand post- 6 months from ACP completion date. This included number of admissions, length of inpatient hospitalisation stay, attendance to the emergency department and specialist outpatient clinics. Total inpatient bill size was used as a marker of healthcare cost. Univariate analysis with paired T-tests was used to explore any significant difference in hospital utilisation rates between pre- and post-ACP in each group.

Results 366 patients and 977 patients completed General ACP and ACP-PPC respectively. For ACP-PPC group, there was significant reduction in healthcare utilisation and cost (\%delta: $36-76 \%, \mathrm{P}<0.05)$. Whereas for General ACP group, the length of stay and inpatient bill size were significantly decreased.

Conclusion ACP forms an integral component in patient care, especially for patients with more advanced diseases. Other than allowing patients to understand personhood and their goals of care, it serves as a platform to moderate healthcare utilisation. This study shows that ACP may reduce healthcare utilisation and cost. 